\title{
WAIS-R Subtest Profile and Cortical Perfusion in Alzheimer's Disease
}

\author{
Gene E. Alexander, 'Isak Prohovnik, Yaakov Stern, \\ AND Richard Mayeux \\ Department of Brain Imaging, New York State Psychiatric Institute and \\ the Departments of Psychiatry, Neurology, and Radiology, College of \\ Physicians and Surgeons, Columbia University, New York, New York
}

WAIS-R profiles were investigated in 28 Alzheimer's disease (AD) and 21 healthy elderly subjects. The Fuld subtest profile, previously reported to have potential as a diagnostic marker for AD, was observed in $35.7 \%$ of our AD patients and $4.8 \%$ of the controls. We compared AD patients with the Fuld profile $(A D F+)$ to a group of patients without the profile $(A D F-$ ) with similar demographics and dementia severity and demographically matched normals using regional Cerebral Blood Flow. Both AD groups showed reduced blood flow in the parietotemporal cortex compared to normals, but the ADF + patients had greater flow reductions than the ADF - group. Examination of WAIS-R performance indicated that the ADF + group had lower scores than the ADF - patients on the Digit Symbol and Block Design subtests, and further, that these two subtests were associated with the parietotemporal perfusion deficit in our AD sample. Our findings do not support the use of the Fuld profile as a diagnostic marker for AD. but do provide physiological evidence for behavioral heterogeneity among $A D$ patients based on WAIS-R subtest performance. 1994 Academic Press. Inc.

The Wechsler Adult Intelligence Scale-Revised (WAIS-R) is often used in the assessment of cognitive decline in dementia. It has been suggested that performance on the individual subtests of the WAIS-R may be specifically useful in aiding the diagnosis of Alzheimer's disease (AD). Fuld (1984) proposed a specific pattern of subtest scores on the

This research was supported in part by NIH Grants AG 05433 and AG 08702 , and grants from the Jean and Louis Dreyfus Foundation and the Charles S. Robertson Memorial Gift for Research in Alzheimer's Disease. An early version of this study was presented at the 19th Annual Meeting of the International Neuropsychological Society, February 13-16, 1991, San Antonio, TX. Address correspondence and reprint requests to Dr. Prohovnik, Unit No. 72, Psychiatric Institute, 722 W. 168th St., New York, NY 10032.

${ }^{1}$ Present address: Laboratory of Neurosciences, Building 10, Room 6C414, NIA, 9000 Rockville Pike, Bethesda, MD 20892. 
WAIS which was associated with cholinergic dysfunction and may facilitate differential diagnosis in the early stages of $\mathrm{AD}$. Her study documented a WAIS subtest profile which occurred with higher frequency among $\mathrm{AD}$ patients than in patients with non-Alzheimer's type dementia, with $44 \%$ sensitivity and $96 \%$ specificity in discriminating $A D$ from other dementia patients in her sample. Additional evidence of high specificity $(84-99 \%)$ and low sensitivity (7-57\%) of the Fuld profile for AD using subtest scores from both the original and revised (WAIS-R) forms of the WAIS has been subsequently reported with numerous populations (Bornstein \& Share, 1990; Bornstein, Termeer, Longbrake, Heger \& North, 1989; Brinkman \& Braun, 1984; Filley, Kobayashi, \& Heaton, 1987; Heinrichs \& Celinski, 1987; Logsdon, Teri, Williams, Vitiello, \& Prinz, 1989; Ryan \& Paolo, 1989; Satz, Hynd, D'Elia, Van Gorp, Ledbetter, \& Connor, 1987; Satz, Van Gorp, Soper, \& Mitrushina, 1987; Tuokko $\&$ Crockett, 1987).

One proposed explanation for the discrepancy between the specificity and sensitivity of the Fuld profile for AD is that it may be indicative of heterogeneity in this patient population (Bornstein et al., 1989). A growing body of literature has shown support for clinical heterogeneity among patients diagnosed with Alzheimer's Disease (Martin, Brouwers, Lalonde, Cox, Teleska, Fedio, Foster, \& Chase, 1986; Mayeux, Stern, \& Spanton, 1985; Prohovnik, Smith, Sackeim, Mayeux, \& Stern, 1989). The Fuld profile may be associated with a specific subgroup of AD patients, accounting for the relatively low sensitivity but high specificity.

Measurement of regional cerebral blood flow (rCBF) using the ${ }^{133}$ xenon inhalation method provides a sensitive and specific measure useful in the discrimination of even early AD from other types of dementia (Prohovnik, Mayeux, Sackheim, Smith, Stern, \& Alderson, 1988; Risberg \& Gustafson, 1988). Further, this rCBF procedure has provided some support for the presence of subgroup differences within the AD population by demonstrating greater degenerative processes occurring in AD patients with presenile compared to senile age at onset (Prohovnik et al., 1989). Preliminary support for the use of the WAIS-R in examining behavioral correlates of $\mathrm{rCBF}$ specific to $\mathrm{AD}$ has also been reported, where several WAIS-R subtests were shown to be predictive of cortical flow deficits in a sample of AD patients (Mayeux, Stern, \& Prohovnik, 1989). An examination of the Fuld profile and its subtests in relation to measures of regional cerebral blood flow would provide a unique opportunity to assess the physiological implications of this WAIS-R subtest profile for $\mathrm{AD}$. The current study was aimed at elucidating the relationship between the Fuld WAIS-R profile and its component subtests to Alzheimer's Disease, using clinical characterization and $\mathrm{rCBF}$ as a marker of pathophysiological severity. 


\section{METHOD}

Subjects. Twenty-eight patients with a clinical diagnosis of probable Alzheimer's Disease according to NINCDS-ADRDA (McKhann, Drachmann, Folstein, Katzman, Price, \& Stadlan, 1984) criteria were accepted into a longitudinal rCBF study. Each patient had a physical and neurological examination with blood work-up (CBC, SMA 20), neuropsychological testing, the Blessed Dementia Rating Scale (BDRS; Blessed, Tomlinson, \& Roth, 1968), and a modified version of the Folstein (Folstein, Folstein, \& McHugh, 1981) Mini-Mental Status examination (mMMS, scores ranging from 0 to 57; Mayeux, Stern, Rosen, \& Leventhal, 1981). Estimates of age at onset ( $A A O)$ and duration of illness were obtained during the clinical evaluation from patient/family reports. The normal control group consisted of 21 healthy elderly volunteers, ages 55 and older, with no history of neurological or psychiatric illness. The AD and normal groups did not differ on means and distributions of age, gender, race, handedness, or English as first language. The normal subjects, however, did show a higher mean number of years of education compared to the AD group (see Table 1). Dementia severity was assessed using screening measures of functional capacity (i.e.. BDRS) and global cognitive functioning (i.e., mMMS). Each subject was administered the WAIS-R (Wechsler, 1981) by trained psychometricians.

Profile definition. The Fuld profile was scored with age-corrected subtest scores using the following formula: $A>B>C \leq D ; A>D$. where $A=$ mean of Information and Vocabulary subtests, $B=$ mean of Similarities and Digit Span subtests, $C=$ mean of Block Design and Digit Symbol subtests, and $D=$ Object Assembly subtest score (Fuld, 1984). Subjects who met the formula criteria were termed Fuld-positive, and those who did not were termed Fuld-negative. Age-corrected subtest scores for subjects older than age 74 $(n=12)$ were scored using the oldest available norms (ages 70-74; Wechsler, 1981). These subjects were included in the AD and normal groups to maintain a representative sample of Alzheimer's patients and age-matched controls.

$r C B F$ procedures. $\mathrm{rCBF}$ was measured using the Novo Cerebrograph 32c system (Novo Diagnostic Systems, Hadsund. Denmark) with 16 detectors covering each cerebral hemisphere. This method has shown high sensitivity and specificity in discriminating AD from other forms of dementia and normal aging (Prohovnik et al.. 1988; Prohovnik. Alexander. Tatemichi, Favate, \& Mayeux, 1991; Prohovnik, Alexander, Tatemichi, \& Mayeux, 1991: Risberg \& Gustafson, 1988). Detector placement reproducibility across subjects was achieved using light markers aligned with the auditory meatus and orbitomeatal line. Extensive procedures for quality control of the $\mathrm{rCBF}$ measurements, previously described in detail (see Prohovnik, 1988), were employed. All rCBF procedures were performed with the subject's eyes closed in a darkened and quiet room. Clearance curves were monitored for a period of $11 \mathrm{~min}$ following a 1 -min administration of ${ }^{131}$ xenon by inhalation. The six-unknown model (M2) was used to analyze the rCBF clearance curves for the Initial Slope Index (1SI: Prohovnik, Knudsen, \& Risberg, 1985; Schroeder, Holstein, Lassen, \& Engell, 1986). The M2 method has been shown to have greater reliability for results in low flow conditions typically seen in dementia (Prohovnik. Brawanski, Pavlakis, Raymond, Lucas, \& Maximillian, 1987; Prohovnik et al.. 1983; Prohovnik et al., 1988; Schroeder et al., 1986). One AD patient was excluded from the $\mathrm{rCBF}$ analyses due to poor data quality. Statistical analyses. Differences between groups for the demographic and clinical variables were compared using analysis of variance and $\chi^{2}$ tests where appropriate. Significant ANOVAs on demographic variables were followed with unpaired $t$ tests (two-tailed) to examine pairwise group differences. Overall group effects for the WAIS-R IQ and ageadjusted subtest scores were compared with analysis of variance followed by pair-wise comparisons using the Newman-Keuls procedure. Analysis of global cerebral blood flow data was conducted using analysis of covariance with the mean ISI value serving as the dependent variable and relevant demographic variables (i.e., age, education) entered first 
TABLE I

Patient and Control Subject Characteristics

\begin{tabular}{|c|c|c|c|}
\hline & nd Control Subjec & acteristics & \\
\hline & & & \\
\hline Variable & $\begin{array}{l}\text { AD patients } \\
(n=28)\end{array}$ & $\begin{array}{l}\text { Normal } \\
\text { controls } \\
(n=21)\end{array}$ & $p$ value \\
\hline Age (years) & $68.6(10.2)$ & $63.4(8.5)$ & ns \\
\hline Education (years) & $11.1(4.1)$ & $14.4(2.8)$ & .003 \\
\hline Gender (frequency) & $\begin{array}{c}\text { Male }=8 \\
\text { Female }=20\end{array}$ & $\begin{array}{c}\text { Male }=6, \\
\text { Female }=15\end{array}$ & ns \\
\hline Race ( $\%$ Caucasian) & 96.4 & 95.2 & ns \\
\hline Handedness (frequency) & $\begin{array}{c}\mathrm{R}=24, \mathrm{~L}=3, \\
\mathrm{~A}=1\end{array}$ & $\begin{array}{c}\mathrm{R}=18 . \mathrm{L}=3, \\
\mathrm{~A}=0\end{array}$ & ns \\
\hline $\begin{array}{l}\text { English as first language } \\
\text { (frequency) }\end{array}$ & $\begin{aligned} \text { Yes } & =19 \\
\text { No } & =9\end{aligned}$ & $\begin{aligned} \text { Yes } & =17 \\
\text { No } & =4\end{aligned}$ & ns \\
\hline mMMS & $31.5(8.2)$ & $54.4(1.7)$ & .0001 \\
\hline BDRS (total) & $9.4(3.8)$ & $0.3(0.5)$ & .0001 \\
\hline WAIS-R FSIQ & $75.3(11.4)$ & $114.7(16.9)$ & .0001 \\
\hline Global ISI & $\begin{array}{c}42.5(8.1) \\
n=27\end{array}$ & $48.3(8.0)$ & .02 \\
\hline
\end{tabular}

Note. mMMS. Modified Mini-Mental Status examination: BDRS. Blessed Dementia Rating Scale; WAIS-R FSIQ. WAIS-R Full Scale IQ; R, right-handed: L, left-handed; A, ambidextrous: Global ISI, rCBF M2 Initial Slope Index value averaged across 32 detectors; ns, not significant. The rCBF values for one AD patient was excluded due to poor data quality.

as covariates. Group differences in the regional pattern of blood flow were initially investigated using a series of repeated-measure ANOVAs for the $16 \mathrm{rCBF}$ detectors, treating hemisphere (right vs. left) as a repeated-measure factor. Since no significant group $x$ hemisphere interactions were observed, detectors were subsequently collapsed across hemispheres and analyzed using ANCOVAs with age and education as covariates. In the 3-group $\mathrm{rCBF}$ analyses. significant overall group effects for the whole cortex means and regional detector values were followed by a series of pairwise ANCOVAs covarying the effects of relevant demographic (i.e., age, education) and clinical variables (i.e., AAO, duration of illness). Bonferroni-adjusted $p$ values were used for all ANCOVA pair-wise comparisons to maintain an overall significance level of $p<.05$. Logistic regression analysis was performed to identify those variables which predicted the occurrence of the Fuld profile in our AD sample. Partial correlations and multiple regression analyses were used to examine the relationship between the WAIS-R subtests and specific rCBF values for the whole AD sample.

\section{RESULTS}

There was a significantly greater prevalence of Fuld profiles in the AD group ( 10 of 28 ) compared to normals ( 1 of $21 ; \chi_{(1, n=49)}^{2}=4.95, p<.03$ ), corresponding to $35.7 \%$ sensitivity and $95.2 \%$ specificity for the Fuld profile in discriminating $\mathrm{AD}$ from normal subjects in this sample. As 
TABLE 2

Comparison of Demographics and Clinical Variables for Fuld Profile Positive and Negative AD Groups

\begin{tabular}{|c|c|c|c|}
\hline \multirow[b]{2}{*}{ Variable } & \multicolumn{2}{|c|}{ Mean $(S D)$} & \multirow[b]{2}{*}{$p$ value } \\
\hline & $\begin{array}{l}\mathrm{ADF}+ \\
(n=10)\end{array}$ & $\begin{array}{l}\mathrm{ADF}- \\
(n=18)\end{array}$ & \\
\hline Age (years) & $62.2(6.3)$ & $72.2(10.3)$ & .01 \\
\hline Education (years) & $13.8(3.8)$ & $9.7(3.6)$ & .01 \\
\hline Gender (frequency) & $\begin{array}{c}\text { Male }=6 \\
\text { Female }=4\end{array}$ & $\begin{array}{c}\text { Male }=2 \\
\text { Female }=16\end{array}$ & .02 \\
\hline Race (\% Caucasian) & 100.0 & 94.4 & ns \\
\hline Handedness (frequency) & $\begin{array}{c}\mathbf{R}=10, \mathrm{~L}=0 . \\
\mathrm{A}=0\end{array}$ & $\begin{array}{c}\mathrm{R}=14 . \mathrm{L}=3 . \\
\mathrm{A}=1\end{array}$ & ns \\
\hline $\begin{array}{l}\text { English as first language } \\
\text { (frequency) }\end{array}$ & $\begin{aligned} \text { Yes } & =10 \\
\text { No } & =0\end{aligned}$ & $\begin{aligned} \text { Yes } & =9 \\
\text { No } & =9\end{aligned}$ & .02 \\
\hline Age at onset (frequency) & $\begin{array}{c}\text { Presenile }=9 \\
\text { Senile }=1\end{array}$ & $\begin{array}{l}\text { Presenile }=7 \\
\text { Senile }=11\end{array}$ & .03 \\
\hline Age at onset (years) & $58.2(7.2)$ & $68.7(10.6)$ & .01 \\
\hline Duration of illness (years) & $4.2(2.8)$ & $3.6(1.7)$ & ns \\
\hline BDRS (total) & $9.8(2.8)$ & $9.3(4.3)$ & ns \\
\hline mMMS & $32.7(7.0)$ & $30.9(8.6)$ & ns \\
\hline WAIS-R FSIQ & $73.2(6.9)$ & $76.4(13.3)$ & ns \\
\hline
\end{tabular}

Note. ADF +, Fuld-positive AD patients: ADF - , Fuld-negative AD patients; mMMS, Modified Mini-Mental Status examination; BDRS, Blessed Dementia Rating Scale; WAIS-R FSIQ, WAIS-R Full Scale IQ; R, right-handed; L, left-handed; A, ambidextrous; Presenile $=$ age at onset $<$ age $65 ;$ Senile $=$ age at onset $\geq$ age $65 ;$ ns, not significant.

indicated in Table 2, the Fuld-positive $\mathrm{AD}(\mathrm{ADF}+)$ patients showed a significantly earlier AAO with a higher proportion of patients falling in the presenile (onset $<$ age 65 ) category ( 9 of 10 ) compared to the Fuldnegative $A D(A D F-$ ) patients ( 7 of 18 ), but the groups did not differ in duration of illness or dementia severity (i.e., BDRS, mMMS). The $\mathrm{ADF}+$ patients were significantly younger and better educated than the ADF - group and differed in the distribution of gender and English as first language (see Table 2).

In examining group differences in cortical perfusion, one ADF + patient was excluded from the analyses due to poor rCBF data quality. The remaining nine $\mathrm{ADF}+$ patients were compared to a group of $\mathrm{ADF}$ $(n=9)$ and normal control $(n=9)$ subjects. These three groups did not differ significantly on distributions of age, education, race, handedness, and English as first language, but did show a strong trend for differences in gender (see Table 3). The two AD groups did not differ significantly on duration of illness, AAO, and dementia severity (i.e., mMMS, BDRS). The three groups also did not differ on variables which are known to 
TABLE 3

$\mathrm{AD}$ and Control Subject Characteristics for rCBF Comparisons

\begin{tabular}{|c|c|c|c|c|}
\hline & & Mean $(S D)$ & & \\
\hline Variable & $\begin{array}{l}\mathrm{ADF}+ \\
(n=9)\end{array}$ & $\begin{array}{l}\mathrm{ADF}- \\
(n=9)\end{array}$ & $\begin{array}{l}\text { Normal } \\
\text { controls } \\
(n=9)\end{array}$ & $p$ value \\
\hline Age (years) & $60.8(4.7)$ & $66.1(9.1)$ & $61.3(7.9)$ & ns \\
\hline Education (years) & $14.3(3.6)$ & $10.9(3.5)$ & $12.3(2.6)$ & ns \\
\hline Gender (frequency) & $\begin{array}{c}\text { Male }=6 \\
\text { Female }=3\end{array}$ & $\begin{array}{c}\text { Male }=1 \\
\text { Female }=8\end{array}$ & $\begin{array}{c}\text { Male }=4 \\
\text { Female }=5\end{array}$ & .054 \\
\hline Race (\% Caucasian) & 100.0 & 88.9 & 88.9 & ns \\
\hline Handedness (frequency) & $\begin{aligned} \mathbf{R} & =9 \\
\mathbf{L} & =\mathbf{0}\end{aligned}$ & $\begin{array}{l}R=6 \\
L=3\end{array}$ & $\begin{array}{l}R=8 \\
L=1\end{array}$ & ns \\
\hline $\begin{array}{l}\text { English as first language } \\
\text { (frequency) }\end{array}$ & $\begin{aligned} \text { Yes } & =9 \\
\text { No } & =0\end{aligned}$ & $\begin{aligned} \text { Yes } & =7 \\
\text { No } & =2\end{aligned}$ & $\begin{aligned} \text { Yes } & =7 \\
\text { No } & =2\end{aligned}$ & ns \\
\hline Age at onset (years) & $56.8(6.1)$ & $63.0(10.0)$ & - & ns \\
\hline Duration of illness (years) & $4.2(3.0)$ & $3.0(1.6)$ & - & ns \\
\hline mMMS & $34.0(6.0)$ & $33,1(9.3)$ & $54.8(1.0)^{a}$ & .0001 \\
\hline BDRS (total) & $9.8(2.9)$ & $9.2(5.3)$ & $0.4(0.5)^{a}$ & .0001 \\
\hline BP. systolic ( $\mathrm{mm} \mathrm{Hg})$ & $128.0(16.2)$ & $134.2(13.1)$ & $136.9(14.6)$ & ns \\
\hline BP, diastolic $(\mathrm{mm} \mathrm{Hg})$ & $\begin{array}{c}78.5(14.1) \\
n=8\end{array}$ & $77.7(9.6)$ & $84.4(7.8)$ & ns \\
\hline $\mathrm{PeCO}_{2}(\mathrm{~mm} \mathrm{Hg})$ & $37.8(3.7)$ & $36.5(4.4)$ & $38.3(2.0)$ & ns \\
\hline
\end{tabular}

Note. ADF +, Fuld-positive AD patients; ADF - . Fuld-negative AD patients; mMMS, Modified Mini-Mental Status examination; BDRS, Blessed Dementia Rating Scale; WAIS-R FSIQ, WAIS-R Full Scale IQ; R, right-handed; L, left-handed; BP, blood pressure; PeCO, end-tidal $\mathrm{CO}_{2}$ partial pressure; ns, not significant. One ADF + patient's diastolic blood pressure value was not available.

${ }^{a}$ Differs significantly from the two AD groups, unpaired $t$ tests (two-tailed), $p<.001$.

influence $\mathrm{rCBF}$ measurement (e.g., blood pressure, end-tidal $\mathrm{CO}_{2}$ pressure).

Although differences in age and years of education among the groups used for the $\mathrm{CCBF}$ comparisons did not reach statistical significance, the patients in the ADF + group were on average 5 years younger than the ADF - group and averaged approximately 3 years more education. It has been well established that age can have significant effects on brain metabolism and rCBF (Horowitz, Duara, \& Rapoport, 1986; Mamo, Meric, Luft, \& Seylaz, 1983). Further, we have found that AD patients with higher levels of educational achievement tend to show greater reductions in perfusion of the parietotemporal cortex than less-well-educated AD patients of equal dementia severity (Stern, Alexander, Prohovnik, \& Mayeux, 1992). To ensure that observed differences between the groups were not simply a function of these demographic differences, analysis of covariance was used for both global and regional flow analyses with age and education entered first as covariates. 


\section{TABLE 4}

ANCOVA Comparisons for rCBF Mean Global and Percentage Detector Values Collapsed Across Hemispheres for AD and Normal Groups

Mean $(S D)$

$\begin{array}{lccccc}\text { rCBF Values } & \begin{array}{c}\text { ADF }+ \\ (n=9)\end{array} & \begin{array}{c}\text { ADF }- \\ (n=9)\end{array} & \begin{array}{l}\text { Normal } \\ \text { controls } \\ (n=9)\end{array} & F \text { test } & p \text { values } \\ \text { Global ISI } & 39.3(7.0)^{a} & 44.4(7.9) & 50.6(5.9) & 4.79 & .019 \\ \text { \%ISI P1 } & 89.2(4.5)^{a} & 95.6(4.0) & 101.8(3.8)^{r} & 17.39 & .0001 \\ \text { \%ISI P2 } & 90.4(5.8)^{a} & 95.8(2.6)^{b} & 97.9(3.2) & 8.15 & .002 \\ \text { \%ISI T2 } & 97.4(6.8) & 102.3(5.8) & 100.8(2.9) & 3.31 & .055 \\ \text { \%ISI T3 } & 99.7(4.6) & 97.8(5.2) & 94.6(2.8) & 3.92 & .035 \\ \text { \%ISI O2 } & 102.9(2.5)^{a} & 98.8(3.9)^{b} & 96.4(3.1) & 13.89 & .0001 \\ \text { PI Index } & 0.86(.06)^{a} & 0.95(.06) & 1.01(.03)^{r} & 13.25 & .0001\end{array}$

Note. ADF +, Fuld-positive AD patients; ADF - , Fuld-negative AD patients; P1-P2, rCBF detectors in the parietal region; T2-T3, rCBF detectors in the temporal region; $\mathrm{O} 2$, rCBF detector in the occipital region; \%ISI, Percentage of whole cortex mean Initial Slope Index values; PI Index, ISI Index expressing parietotemporal perfusion (PI and P3) divided by superior Rolandic (C1) and occipital (O2) regions. ANCOVAs were used to compute overall $F$ values with age and education entered first as covariates. Follow-up pair-wise comparisons used Bonferroni-adjusted $p$ values for ANCOVAs controlling for age and education. Age at onset and duration of illness were added as covariates for comparisons between the two AD groups.

a Significant difference after controlling for age and education, ADF + vs. Normals.

b Significant difference after controlling for age, education, AAO, and duration of illness, ADF + vs. ADF - .

${ }^{r}$ Significant difference after controlling for age and education, ADF - vs. Normals.

After controlling for the effects of age and education, the three groups differed significantly on mean ISI for whole cortex (see Table 4). Education was a significant covariate for the mean ISI value $\left(F_{(1,22)}=5.60, p\right.$ $<.03$ ), while no significant covariate effects for age were found. Pair-wise comparisons for mean ISI were conducted using ANCOVAs with age and education initially entered as covariates for group comparisons with normals. Since AAO and duration of illness can also account for reductions in $\mathrm{rCBF}$, these two clinical variables were additionally entered as covariates for the comparison of the two AD groups. The pairwise analyses indicated that the ADF + patients had significantly reduced mean flow compared to the normals, but did not differ from the ADF - group. When compared to the normal controls, the ADF - patients also showed a lower mean flow but this difference did not reach significance ( $p=$ .057).

Individual detector values are confounded by subject differences in perfusion levels for whole cortex. To examine regional patterns independent of overall level, values for each detector were divided by the whole 
cortex means for each subject. The resulting values (\%ISI) were each initially analyzed for the $16 \mathrm{rCBF}$ detector regions using repeatedmeasure ANOVAs with group (ADF + vs. ADF - vs. normals) as the between-subject and hemisphere (left vs. right) as the repeated-measure factors.

Since no group by hemisphere interactions were found, \%ISI means were subsequently collapsed across hemisphere. As shown in Table 4, ANCOVAs with \%ISI values showed significant group effects for two of the four parietal detectors (P1 and P2) after initially controlling for the effects of age and education. In addition, significant main effects for group were present for detectors in the occipital (O2) and temporal (T3) regions with a trend for an additional temporal lobe detector (T2). No group differences with \%ISI scores for other detectors were observed and no significant covariate effects for age or education were found for those detectors showing significant group differences. Pairwise comparisons using ANCOVAs with Bonferroni-adjusted $p$ values indicated that, after controlling for age and education, both $\mathrm{AD}$ groups had reduced flow in the parietal region (PI) compared to normals. However, the ADF + patients also had reduced blood flow in an additional parietal detector $(\mathrm{P} 2)$ and relatively increased flow in the occipital $(\mathrm{O} 2)$ region compared to normals. Compared to the ADF - patients, the ADF + group had greater blood flow reductions in the parietal lobes $(\mathrm{P} 2)$ and relatively increased flow in the occipital region (O2), after additionally controlling for AAO and illness duration. Pair-wise group differences for a detector in the temporal region (T3) did not reach significance with the Bonferroni adjustment, but there was a strong trend $(p=.022)$ for the ADF + patients having a relatively higher flow than normals. The group differences in the pattern of \%ISI scores across the $16 \mathrm{rCBF}$ detector regions are depicted in Fig. 1.

In addition, the groups were compared with an index of parietotemporal deficit using ISI perfusion values of the parietotemporal cortex (PI and P3) divided by the superior Rolandic (C1) and occipital pole (O2) regions. This index of parietotemporal perfusion has been shown to discriminate $\mathrm{AD}$ patients from normals and non-Alzheimer's dementia patients (Prohovnik et al., 1991). After entering age and education first as covariates, a significant ANCOVA effect for group was observed with this parietotemporal index (see Table 4). Pair-wise ANCOVAs with Bonferroni adjustment indicated that both AD groups had significantly lower index scores than the normals after controlling for age and education. The ADF + group also had a lower index score than the ADF - patients, but this difference did not reach statistical significance $(p=.058)$ after entering age, education, AAO, and duration of illness as covariates.

Group differences on the WAIS-R IQ and subtest scores for those subjects used in the rCBF comparisons are shown in Table 5. ANOVAs 


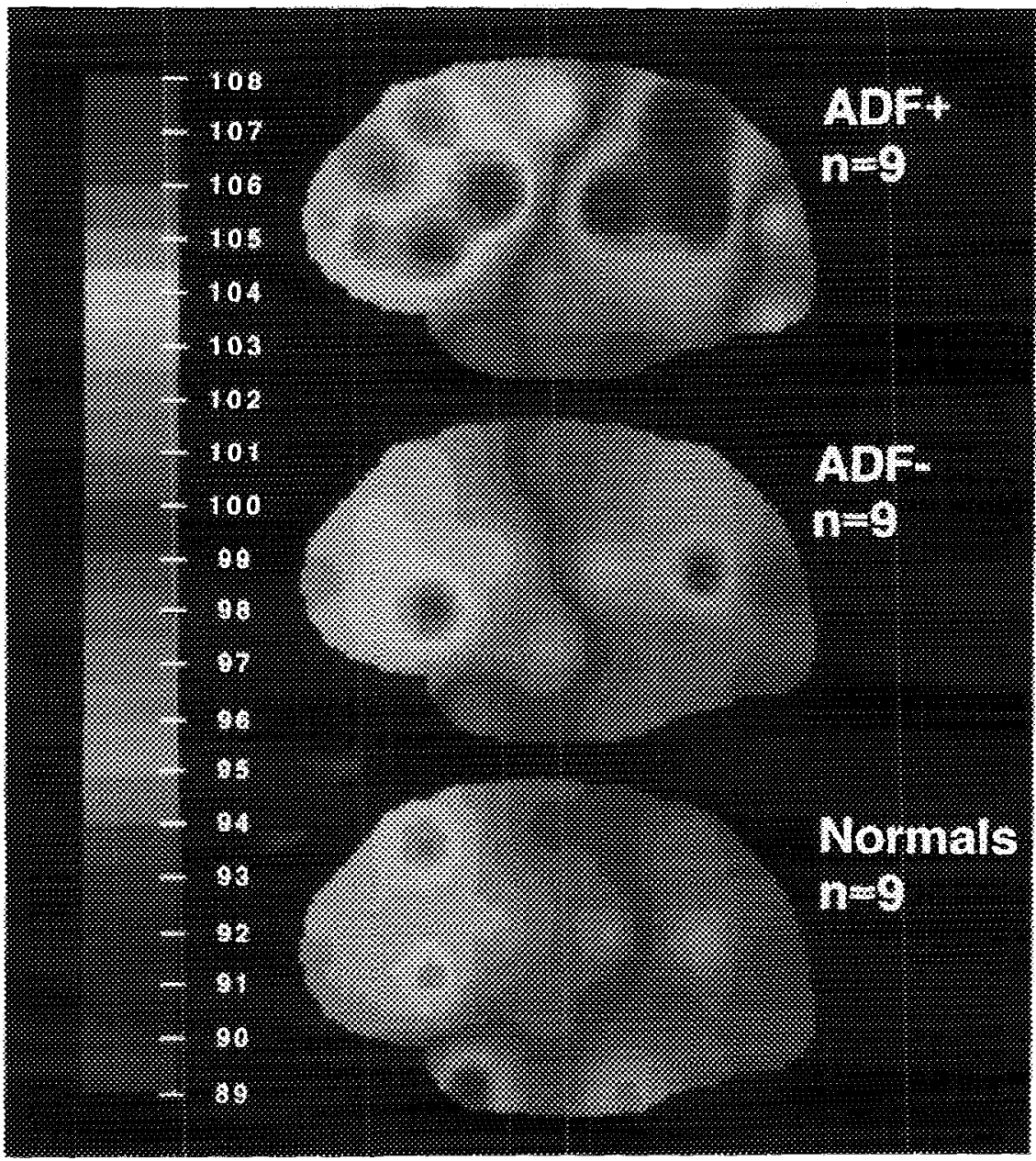

Fig. 1. Comparison of regional perfusion for the AD and normal groups. $\mathrm{rCBF}$ is expressed as a percentage of whole cortex mean flow (ISI) at each detector and averaged across right and left hemispheres. Red on the color scale indicates relatively higher cortical perfusion values, while the blue end of the scale represents lower flows. Note the relative flow reductions for both $\mathrm{AD}$ groups in the parietotemporal region compared to those of the normals with the ADF + patients showing a more extensive parietotemporal deficit than the ADF - group. rCBF, regional cerebral blood flow; 1S1, initial slope index parameter using the six unknown model; AD, Alzheimer's disease; ADF + Fuld-positive AD patients; Fuld-negative AD patients. 
TABLE 5

Mean WAIS-R IQ and Age-Adjusted Subtest Scores for AD and Normal Groups Selected for the rCBF Comparisons

\begin{tabular}{|c|c|c|c|c|c|}
\hline & & Mean $(S D)$ & & & \\
\hline WAIS-R Scores & $\begin{array}{l}\mathrm{ADF}+ \\
(n=9)\end{array}$ & $\begin{array}{l}\text { ADF - } \\
(n=9)\end{array}$ & $\begin{array}{l}\text { Normals } \\
(n=9)\end{array}$ & $F$ test & $p$ value \\
\hline Full Scale IQ & $74.3(6.3)$ & $78.6(16.7)$ & $106.4(10.3)^{a}$ & 19.33 & .0001 \\
\hline Verbal IQ & $81.8(8.2)$ & $83.3(14.8)$ & $110.1(13.1)^{\prime \prime}$ & 14.91 & .0001 \\
\hline Performance IQ & $65.9(4.0)$ & $74.8(16.8)$ & $99.8(6.8)^{4}$ & 24.15 & .0001 \\
\hline Information & $8.0(2.3)$ & $6.2(2.9)$ & $12.1(2.3)^{i i}$ & 12.76 & .0002 \\
\hline Digit Span & $6.7(2.2)$ & $8.3(3.8)$ & $11.3(2,1)^{4}$ & 6.43 & .006 \\
\hline Vocabulary & $8.6(2.8)$ & $8.4(2.5)$ & $12.6(2.6)^{4}$ & 7.11 & .004 \\
\hline Arithmetic & $5.0(1.3)$ & $5.7(3.6)$ & $11.0(2.6)^{17}$ & 13.58 & .0001 \\
\hline Comprehension & $5.4(2.0)$ & $7.2(4.1)$ & $12.6(2.2)^{\mathrm{th}}$ & 14.50 & .0001 \\
\hline Similarities & $6.3(1.9)$ & $6.7(2.7)$ & $10.8(3.1)^{4}$ & 8.00 & .002 \\
\hline Picture Completion & $5.0(1.9)$ & $5.2(3.7)$ & $11.0(1.7)^{11}$ & 15.47 & .0001 \\
\hline Picture Arrangement & $3.8(1.9)$ & $5.7(3.5)$ & $11.1(1.7)^{\prime \prime}$ & 20.82 & .0001 \\
\hline Block Design & $2.8(1.3)^{h}$ & $5.3(3.5)$ & $10.6(1.7)^{a}$ & 25.38 & .0001 \\
\hline Object Assembly & $4.9(1.4)$ & $5.3(3.7)$ & $8.7(1.8)^{\prime \prime}$ & 6.19 & .007 \\
\hline Digit Symbol & $1.9(1.2)^{h}$ & $5.1(4.2)$ & $9.9(1.8)^{\prime \prime}$ & 19.69 & .0001 \\
\hline
\end{tabular}

Note. ANOVAs were used for overall group comparisons followed by the NewmanKeuls procedure for multiple pairwise comparisons. $p<.05$.

"Differs significantly from both AD groups.

"Differs significantly from the ADF - group.

revealed significant overall group effects for all IQ and age-adjusted subtest scores. Pairwise comparisons using the Newman-Keuls procedure indicated that the normals scored higher than both AD groups on the IQ and subtest scores. In addition, the ADF + group showed poorer performance on the Block Design and Digit Symbol subtests compared to the ADF - patients (see Fig. 2). No differences between the AD groups on other subtest or IQ scores were observed. To further examine the differences between the two AD groups on the Block Design and Digit Symbol subtests analysis of covariance was performed. ANCOVAs with education, AAO, and duration of illness entered first as covariates reduced the group effects to nonsignificance for both subtests. However, a significant covariate effect for AAO was found for both the Block De$\operatorname{sign}\left(F_{(1.13)}=23.21, p<.0001\right)$ and Digit Symbol $\left(F_{(1.13)}=24.47, p<\right.$ $.0001)$ subtest comparisons.

Since several demographic, clinical severity, WAIS-R subtest, and perfusion variables differed significantly between the $\mathrm{AD}$ patients with and without the Fuld profile, we performed a logistic regression analysis to determine which factors are most important in contributing to the presence of the Fuld profile in AD. A step-wise logistic regression analysis for the total sample of AD patients $(n=27)$ was used with the presence 


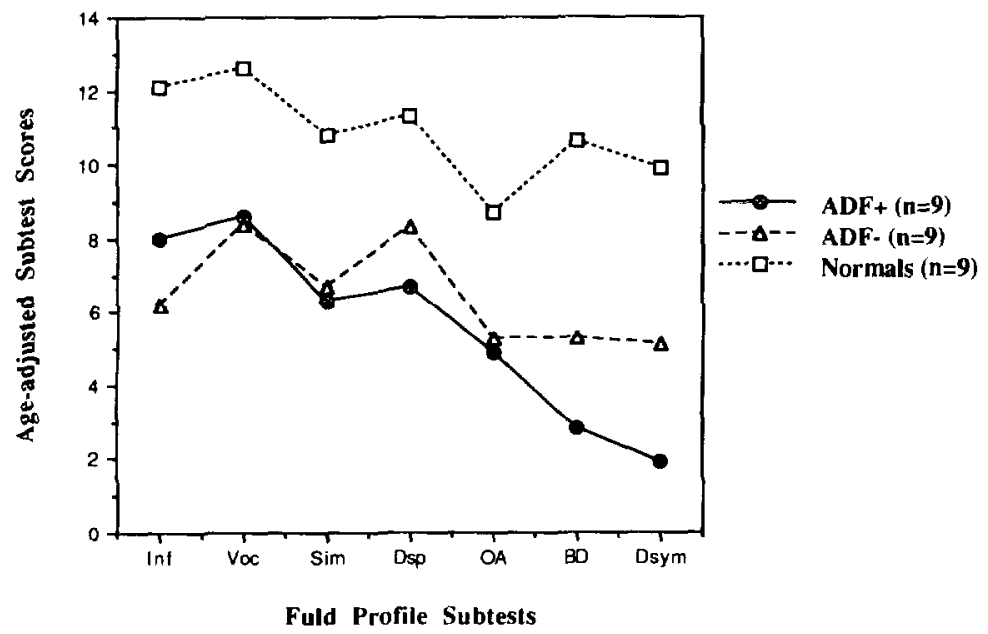

Fig. 2. Age-adjusted WAIS-R Fuld profile subtests for the AD and normal control groups used in the $\mathrm{rCBF}$ comparisons. Both AD groups scored lower than normals across all subtests, but the ADF + patients differed significantly from the ADF - group on the Block Design and Digit Symbol subtests. Inf, Information; Voc, Vocabulary; Sim, Similarities; Dsp, Digit Span; OA, Object Assembly; BD, Block Design; Dsym, Digit Symbol; ADF+. Fuld-positive AD patients; ADF-, Fuld-negative AD patients.

or absence of the Fuld profile as the dependent variable and relevant demographics (i.e., age, education, gender, English as the first language), clinical variables (i.e., AAO, duration, mMMS, BDRS), WAIS-R subtests (i.e., Digit Symbol, Block Design), and rCBF values (i.e., global ISI, \% P1, \%P2, parietotemporal index) as independent variables. The analysis produced a model accurately classifying $92.6 \%$ of our AD patients as with or without the Fuld profile using three selected variables. The best predictor of the Fuld profile was the interaction of age $\times$ parietotemporal index $\left(\chi_{(1, n=27)}^{2}=15.05, p<.0001\right)$ followed by gender $\left(\chi_{(1, n-27)}^{2}=7.42, p<.006\right)$ and the \%ISI P2 detector value $\left(\chi_{(1, n=27)}^{2}=\right.$ $3.86, p<.05$ ), respectively.

To investigate the relationship between AAO and the WAIS-R subtests irrespective of the Fuld profile, a series of partial correlations was performed for the total AD sample $(n=27)$. After controlling for education, duration of illness, and dementia severity (i.e., mMMS, BDRS), AAO was significantly associated with the Digit Symbol $(r=.64, p<.001)$, Block Design $(r=.51, p<.007)$, Arithmetic $(r=.49, p<.01)$, Picture Arrangement $(r=.39, p<.03)$, Picture Completion $(r=.42, p<.02)$, Comprehension $(r=.57, p<.002)$, Vocabulary $(r=.40, p<.03)$, and Similarities $(r=.36, p<.05)$ subtests. AAO was also correlated with the Performance IQ $(r=.53, p<.005)$, Verbal IQ $(r=.48, p<.01)$, and Full Scale IQ $(r=.52, p<.006)$ scores. Since gender was a signifi- 
cant predictor of the occurrence of the Fuld profile among our AD patients, we used unpaired t-tests to assess gender differences on the WAIS-R subtests for the whole AD sample. No significant gender effects were observed for performance on any subtest.

We also conducted partial correlations to examine the association between the WAIS-R subtests and the parietotemporal perfusion deficit in our total AD sample. After controlling for relevant demographic (i.e., education) and clinical severity (i.e., AAO, duration of illness, mMMS, BDRS) variables, the parietotemporal perfusion index was significantly correlated with the Block Design $(r=.37, p<.04)$, Digit Symbol $(r=$ $.54, p<.005)$, Object Assembly $(r=.40, p<.03)$, and Vocabulary $(r=-.42, p<.03)$ subtests. No other WAIS-R subtests were significantly related to the parietotemporal perfusion index. Since the \%ISI P2 value was the parietal flow variable which differed significantly between the two $A D$ groups in our sample, we also examined the relationship between this specific detector and the WAIS-R subtests for the AD patients. After controlling for the same demographic and clinical severity variables, the \%ISI P2 value was significantly correlated with the Digit Symbol $(r=.43, p<.02)$ subtest. No other subtests were significantly associated with this detector value.

We entered the significant WAIS-R subtests into multiple regression analyses to assess the best predictors of the parietotemporal perfusion index and \%ISI P2 detector values in our whole AD sample. After initially entering AAO, education, duration of illness, BDRS, and mMMS into the regression equations, the Digit Symbol subtest was the single best predictor of the parietotemporal perfusion index, accounting for a significant incremental increase of $16.2 \%$ of the variance $\left(F_{(1,19)}=8.31, p<\right.$ $.009)$. However, no subtests were able to significantly predict the \%ISI $P 2$ value.

\section{DISCUSSION}

Several studies investigating the occurrence of the Fuld profile among $A D$ patients have described it as a potential diagnostic marker in the early stages of Alzheimer's Disease. However, our results, consistent with previous findings, support the high specificity but low sensitivity of the Fuld profile for AD. In the current sample, approximately $36 \%$ of a representative group of $\mathrm{AD}$ patients presented with the Fuld profile, whereas $5 \%$ of the normal controls were Fuld-positive. This well replicated finding may be indicative of behavioral heterogeneity within the AD population; or it may simply be a function of the psychometric limitations of the profile, including its potential vulnerability to demographic differences among AD patients. The current investigation sought to examine the Fuld profile and its component WAIS-R subtests in terms of 
rCBF and clinical variables for a sample of $\mathrm{AD}$ patients and healthy elderly controls.

\section{Clinical Characterization and Prevalence of the Fuld Profile}

Our ADF + patients had an early age at onset. In our sample, there was a higher prevalence of the Fuld profile among presenile (onset $<$ age 65) AD patients ( 9 of 16) than for senile onset patients ( 1 of 12) and normals ( 1 of 21). In contrast, the ADF + and ADF - groups did not appreciably differ in duration of illness, nor were they different in the severity of dementia as assessed by screening measures of both functional (i.e., BDRS) and global cognitive (i.e., mMMS) abilities.

The ADF + patients were younger and had more years of education than the ADF - group, and also differed on the distributions of gender and English as first language. Since age-corrected subtest scores were used in scoring the Fuld profile, it is not expected that the observed age difference alone could account for the occurrence of the Fuld profile in our AD patients. However, education and English as first language have been described as variables which may affect performance on intellectual measures (Kaufman, 1979) and specifically the Fuld profile (Fuld, 1986). The AD groups did not differ on WAIS-R FSIQ, but higher levels of education might be required for a patient to demonstrate the relative pattern of subtest performances which defines the presence of the profile. For example, it is possible that individuals with less education may be less likely to demonstrate relatively higher Information and Vocabulary subtest scores due to less premorbid educational experience. In a study examining the base rates of the Fuld profile in the WAIS- $R$ standardization sample, Satz and colleagues (1990) reported an increased prevalence of the profile, ranging from 12 to $15 \%$, among elderly normals with greater than high school level educations. However, the profile occurred at two to three times this base rate among our AD patients ( $35.7 \%$ ). Thus, educational differences alone do not seem sufficient to explain the prevalence of the profile in our $\mathrm{AD}$ sample.

In our sample, no subjects without English as a first language were found in the ADF + group. Performance on tests of intellectual ability may underestimate levels of functioning, especially in the areas of verbal skills, for those subjects whose first language is not English (Kaufman, 1979). In the case of the Fuld profile, AD patients whose first language is other than English may also be less able to produce the pattern of relative subtest performances needed to obtain the profile. The AD patients in this sample who have less educational experience were not necessarily those with other than English as a first language. A comparison of ADF - patients with and without English as a first language show no difference in years of education with both groups having a mean of 10 and 9 years, respectively. 
An effect for gender has also been reported among normals in the WAIS-R standardization sample (Satz et al., 1990), with a small but higher prevalence of the Fuld profile occurring among males than females (7 vs. $5 \%$, respectively). In our $\mathrm{AD}$ sample $75 \%$ (6 of 8 ) of the males demonstrated the Fuld profile, while only $20 \%$ (4 of 20) of the females were Fuld-positive, suggesting a gender effect which far exceeds what has been previously reported among normals. However, there were no significant gender effects or group by gender interactions when the $\mathrm{ADF}+$ and ADF - groups were compared on the individual WAIS-R subtest scores. This suggests that the observed gender effect in our sample is associated with the relative pattern of the Fuld profile and not the magnitude of performance on the individual subtests. The gender effect is also not explained by differences in years of education, age, AAO, or English as first language, as there were no significant gender effects or group by gender interactions for these variables. Thus, gender seems to be an important factor related to the prevalence of the Fuld profile in our sample, but it is not related to differences in levels of performance on the WAIS-R subtests or other demographic characteristics.

Overall, these findings suggest that in terms of clinical and demographic characteristics for this sample of Alzheimer's patients, the Fuld profile identified a group of relatively younger $A D$ patients with an early age at onset, and who have a relatively higher level of education, on average completing 1-2 years above the high school level. Further, this group included more males compared to those without the profile and excluded patients who did not learn English as their first language. However, the Fuld profile was not related to estimates of illness duration or overall dementia severity.

\section{Cortical Perfusion and WAIS-R Subtest Profiles}

In investigating the implications of the Fuld profile for $\mathrm{rCBF}$, ADF + patients were compared to a group of ADF - patients with similar demographics and dementia severity, as well as a group of demographically matched normals. The ADF + patients presented with a prominent pattern of reduced cortical blood flow, including flow reductions in the parietotemporal region with relative increases in the occipital region compared to normals. The ADF - patients also showed reduced blood flow in the parietotemporal cortex, but the $\mathrm{ADF}+$ group clearly demonstrated more extensive parietal lobe reductions as well as a marked global decrease in flow. Thus, both $\mathrm{AD}$ groups showed regional patterns of blood flow consistent with those typically seen in Alzheimer's disease; but the $\mathrm{ADF}+$ group demonstrated a more extreme variant.

To ensure that our Fuld profile group findings for rCBF were not simply a function of differences on relevant demographic and clinical variables, 
we used analysis of covariance for the group comparisons with age, education, AAO, and duration of illness entered first as covariates. Thus, our findings which show greater perfusion deficits among Alzheimer's patients who demonstrate the Fuld profile than those who don't are not likely to be the result of differences among the groups on these demographic and clinical variables. The two AD groups used in the $\mathrm{rCBF}$ comparisons also differed in the distribution of gender, with mostly female patients in the ADF - group. Some studies have reported gender differences in cortical perfusion, where healthy young females showed higher resting flows than males (Gur, Gur, Obrist, Hungerbuhler, Younkin, Rosen, Skolnick, \& Reivich, 1982; Shaw, Meyer, Mortel, Cutaia, Sakai, Yamaguchi, \& Amamoto, 1979). Although our sample size is small, an investigation of gender differences for global $\mathrm{rCBF}$ and parietotemporal perfusion values among our elderly subjects revealed no significant effects for gender or group by gender interactions.

Examination of group differences for the WAIS-R subtests revealed that both $\mathrm{AD}$ groups used in the $\mathrm{CCBF}$ comparisons showed poorer performance than normals across all IQ and subtest scores. The ADF + patients also showed significantly poorer performance on the Block Design and Digit Symbol subtests compared to the ADF - group. This finding indirectly suggests that it may not be the Fuld profile which is specifically associated with the greater parietotemporal deficit, but rather the poorer performance on these two component subtests. When we examined the relationship between the partietotemporal perfusion index and the WAIS-R subtests for the whole AD sample, we found several Performance subtests, including Block Design and Digit Symbol to be significantly related to this perfusion index. Further, the Digit Symbol subtest was the single best predictor of the parietotemporal perfusion deficit, accounting for an increase of $16.2 \%$ of the variance over and above the effects of relevant demographic and dementia severity variables, including $\mathrm{AAO}$. Together, these findings suggest that the greater parietotemporal perfusion deficit observed among our $\mathrm{ADF}+$ patients may be more specifically related to the magnitude of poor performance on the component subtests assessing visuospatial-motor skills than simply the relative pattern defined by the Fuld profile.

That the two AD groups differed on the rCBF deficit and the Block Design and Digit Symbol subtests, but not on overall dementia severity (i.e., mMMS, BDRS), suggests that these measures of visuospatialmotor functions may be more sensitive to the neurophysiological deficit characteristic of $\mathrm{AD}$ than global measures of cognitive and functional abilities. Deficits involving visuospatial tasks have been associated with functioning in the parietal brain regions (Damasio, 1988; Fried, Mateer, Ojemann, Wohns, \& Fedio, 1982). Our findings show support for this functional relationship with a positive correlation between these two 
WAIS-R Performance subtests and the parietotemporal perfusion deficit. Thus, in addition to the diffuse clinical decline associated with dementia, we have shown support for specific areas of cognitive deficit corresponding to regional areas of brain dysfunction characteristic of AD. Further, Digit Symbol has been described as the WAIS-R subtest most sensitive to the effects of brain injury (Lezak, 1983). This finding was supported in our sample of AD patients with Digit Symbol being the single best predictor of the parietotemporal deficit among the WAIS-R subtests.

Additional analyses examining the difference between $\mathrm{ADF}+$ and ADF - groups on the Block Design and Digit Symbol subtests revealed that these effects (but not the $\mathrm{rCBF}$ differences) were reduced to nonsignificance when we controlled for AAO. Thus, the relatively poorer performance of the ADF + patients on the two WAIS-R subtests may be in part related to the earlier AAO observed in the ADF + group. Partial correlations examining how AAO relates to WAIS-R subtest scores for the total AD sample supported this finding. After controlling for education, duration of illness, and dementia severity, AAO was significantly associated with several WAIS-R subtests, including Block Design and Digit Symbol. Age at onset has not been previously described as a factor associated with patterns of WAIS-R performance in AD, but greater levels of overall cognitive impairment have been reported for Fuld-positive AD patients (Filley et al., 1987). Our findings suggest that the magnitudes of subtest performance should be considered, in addition to the relative patterns, for the WAIS-R profiles to identify meaningful subgroups of AD patients. Since the criteria for the Fuld profile do not take into account the actual magnitude of subtest scores, it seems unlikely that this profile would consistently identify the same subgroup of $A D$ patients observed in our sample.

Overall, our results indicate a specific interrelationship among cognitive performance, demographic variables, and perfusion of the parietotemporal cortex, supporting previous reports of clinical heterogeneity within the $\mathrm{AD}$ population. The current findings suggest that WAIS-R subtests are associated with the specific pattern of cortical perfusion attributed to $\mathrm{AD}$ and may be specifically useful in identifying $\mathrm{AD}$ patients with more severe parietotemporal perfusion deficits in the absence of greater clinical dementia severity. Further, our findings provide additional evidence supporting age at onset as an important factor in identifying subgroup differences among $\mathrm{AD}$ patients. In our $\mathrm{AD}$ sample, poorer WAIS-R subtest performance was associated with earlier age of disease onset after controlling for other demographic and clinical variables.

\section{Conclusion}

Consistent with previous reports, we have found the WAIS-R Fuld profile to demonstrate high specificity but low sensitivity for Alzheimer's 
Disease in our sample of AD patients and normals. The Fuld profile identified a group of patients with a specific pattern of demographic, clinical, and neurophysiological characteristics which distinguished them from the AD patients without the profile. Further examination of the Fuld profile indicated that the observed clinical and neurophysiological differences between our AD groups with and without the profile can be explained by the magnitude of performance on two of the WAIS-R subtests included in the profile, i.e., Block Design and Digit Symbol. Thus, our findings suggest that more important than the relative pattern of the Fuld profile is the magnitude of scores on several Performance subtests of the WAIS-R, especially Digit Symbol. Although the Fuld profile requires relatively poorer performance on subtests assessing visuospatial-motor skills than for those assessing verbal intellectual abilities, the profile does not include criteria to address the degree of relative differences among the subtests. In our sample, the ADF + patients showed significantly poorer performance on the Block Design and Digit Symbol subtests compared to the ADF - group. Other samples of AD patients identified by the Fuld profile may not reliably demonstrate this difference and therefore, may not show the earlier age at onset and greater reductions in parietotemporal perfusion that we observed. By not specifying criteria for the magnitude of differences between the subtests, the Fuld profile is more likely to be susceptible to the effects of chance measurement error in identifying ADF + patients across different samples.

That the Block Design and Digit Symbol subtests were correlated with $\mathrm{AAO}$ and the parietotemporal perfusion deficit characteristic of $\mathrm{AD}$, but were not significantly related to other demographic and clinical variables suggests that poorer visuospatial-motor skills as assessed by WAIS-R subtests is associated with a greater disease severity that is not evident on global clinical measures of dementia. Thus, these subtests and especially Digit Symbol may provide greater sensitivity to the neurophysiological effects of AD and may be specifically useful in identifying a subgroup of AD patients with an earlier AAO and more severe parietotemporal deficits. This subtype variability on specific measures of cognitive performance further support the common clinical observation of behavioral heterogeneity in $\mathrm{AD}$.

The diagnosis of subgroup characteristics in AD may be particularly important given the current interest in developing pharmacological interventions for Alzheimer's patients. Careful subgroup classification in order to develop and evaluate potential treatments which target specific behavioral and pathophysiological changes observed among AD patients may be warranted. The examination of cognitive performance in conjunction with neurobiological measures of brain function, such as $\mathrm{rCBF}$, may be useful in this classification process. Further research examining the relationship between the parietotemporal perfusion deficit, specific cogni- 
tive functions, and the cholinergic deficiency attributed to AD is needed. In addition, the longitudinal implications of subgroup heterogeneity in AD is unclear. Studies to help elucidate the relationship between changes in the severity of the parietotemporal deficit and subgroup differences in cognitive performance, disease progression, and response to therapeutic trials among $\mathrm{AD}$ patients is warranted. These investigations may add to our knowledge concerning the specific behavioral and pathophysiological effects of this disease, and further, may lead to potential advancement in the treatment of $\mathrm{AD}$ patients.

\section{REFERENCES}

Blessed. G., Tomlinson. B. E., \& Roth. M. 1968. The association between quantitative measures of dementia and senile changes in the cerebral grey matter of elderly subjects. British Journal of Psychiatr, 114, 797-891.

Bornstein. R. A.. \& Share. D. 1990. Prevalence of the Fuld profile in temporal lobe epilepsy. Joumal of Clinical and Experimental Neuropsychology, 12, 265-269.

Bornstein, R. A., Termeer, J.. Longbrake, K.. Heger, M., \& North. R. 1989. WAIS-R cholinergic deficit profile in depression. Psychological Assessment: Journal of $\mathrm{Con}$ sulting and Clinical Psyctology. 1, 342-344.

Brinkman. S. D., \& Braun. P. 1984. Classification of dementia patients by a WAIS profile related to central cholinergic deficiencies. Journal of Clinical Neuropsychology. 6, $393-400$.

Damasio. A. R. 1988. Disorders of complex visual processing: Agnosias, achromatopsit, Balint's syndrome, and related difficulties of orientation and construction. In M.-M. Mesulam (Ed.). Principles of behavioral nemology. Philadelphia. PA: Davis. Pp. $259-288$.

Filley. C. M.. Kobayashi. J.. \& Heaton. R. K. 1987. Wechsler intelligence scale profiles. the cholinergic system. and Alzheimer's disease. Journal of Clinical and Experimental Neuropsychology, 9, 180-186,

Folstein, M. F., Folstein, S. E., \& McHugh, P. R. 1981. "Mini-mental S1ate": A practicial method for grading the cognitive state of patients for the clinician. Joumal of Psychiatric Research. 12, 189-198.

Fried. I. Mateer. C.. Ojemann, G.. Wohns, R., \& Fedio, P. 1982. Organization of visuospatial functions in human cortex: Evidence from electrical stimulation. Brain, 105, 349-371.

Fuld, P. A. 1984. Test profile of cholinergic dysfunction and Alzheimer's type dementia. Journal of Clinical Neuropsychology, 6, 380-392.

Fuld. P. A. 1986. Pathological and chemical validation of behavioral features of Alzheimer's disease. In L. W. Poon (Ed.). Handbook for clinical memory assessment of older adults. Washington. DC: American Psychological Association. Pp. 302-306.

Gur, R. C., Gur, R. E., Obrist, W. D., Hungerbuhler, J. P., Younkin, D., Rosen. A. D.. Skolnick, B. E., \& Reivich, M. 1982. Sex and handedness differences in cerebral blood flow during rest and cognitive activity. Science. 217, 659-661.

Heinrichs. R. W.. \& Celinski. M. J. 1987. Frequency of occurrence of a WAIS dementia profile in male head trauma patients. Joumal of Clinical and Experimental Neuropsychology. 9, 187-190.

Horowitz. B.. Duara. R.. \& Rapoport, S. 1. 1986. Age differences in intercorrelations between regional cerebral metabolic rates for glucose. Annals of Nourology, 19, 60-67.

Kaufman. A. S. 1979. Intelligent testing with the WISC-R. New York: Wiley. 
Lezak, M. D. 1983. Neuropsychological assessment. New York: Oxford Univ. Press.

Logsdon, R. G., Teri, L., Williams, D. E., Vitiello, M. V., \& Prinz, P. N. 1989. The WAIS-R profile: A diagnostic tool for Alzheimer's disease? Journal of Clinical and Experimental Neuropsychology, 11, 892-898.

Mamo, H., Meric, P., Luft, A., \& Seylaz, A. 1983. Hyperfrontal pattern of human cerebral circulation. Archives of Neurology, 40, 626-632.

Martin, A., Brouwers, P., Lalonde, F., Cox, C., Teleska, P., Fedio, P., Foster, N. L., \& Chase, T. N. 1986. Towards a behavioral typology of Alzheimer's patients. Journal of Clinical and Experimental Neuropsychology, 8, 594-610.

Mayeux, R., Stern, Y.. \& Prohovnik, I. 1989. Behavior that predicts relative rCBF perfusion in Alzheimer's disease. Neurology (Suppl. 1), 39, 168-169.

Mayeux, R.. Stern, Y.. Rosen. J., \& Leventhal, J. 1981. Depression, intellectual impairment, and Parkinson's disease. Neurology, 31, 645-650.

Mayeux, R., Stern, Y., \& Spanton, S. 1985. Clinical heterogeneity in patients with dementia of the Alzheimer's type. Neurology, 35, 453-461.

McKhann, G., Drachman, D., Folstein, M., Katzman, R., Price, D., \& Stadlan, E. M. 1984. Clinical diagnosis of Alzheimer's disease: Report of the NINCDS-ADRDA work group under the auspices of Department of Health and Human Services Task Force on Alzheimer's disease. Neurology, 34, 939-944.

Prohovnik, I. 1988. Data quality, integrity, and interpretation. In S. Knezevic, V. A. Maximillian. Z. Mubrin, 1. Prohovnik, \& J. Wade (Eds.), Handbook of regional cerebral blood flow. Hillsdale, NJ: Erlbaum. Pp. 51-78.

Prohovnik, I., Alexander. G. E., Tatemichi, T. K., \& Mayeux, R. 1991. Exploring the nature of the parietotemporal perfusion deficit in AD. Journal of Cerebral Blood Flow and Metabolism (Suppl. 2), 11, 179.

Prohovnik, I., Alexander, G. E., Tatemichi. T. K., Favate, A., \& Mayeux, R. 1991. Cortical perfusion in vascular and Alzheimer's dementia. Neurology (Suppl. 1), 41, 358.

Prohovnik, 1., Brawanski, A., Pavlakis, S., Raymond, R., Lucas, L., \& Maximillian, V. A. 1987. Vascular compartment in noninvasive ${ }^{133}$ Xe clearance. Journat of Cerebral Blood Flow and Metabolism (Suppl. 1), 7, 551.

Prohovnik, I., Knudsen, E., \& Risberg, J. 1983. Accuracy of models and algorithms for determination of fast compartment flow by noninvasive 133-Xe clearance. In P. Magistretti (Ed.), Functional radionuclide imaging of the brain. New York: Raven. Pp. $87-115$.

Prohovnik, I., Knudsen, E., \& Risberg. J. 1985. Theoretical evaluation and simulation test of the initial slope index for noninvasive rCBF. In H. Hartmann \& S. Hoyer (Eds.), Cerebral blood flow and metabolism measurement. Berlin: Springer-Verlag. Pp. 56-60.

Prohovnik, I., Mayeux, R., Sackheim, H. A., Smith, G., Stern, Y., \& Alderson, P. O. 1988. Cerebral perfusion as a diagnostic marker of early Alzheimer's disease. Neurology, 38, 931-937.

Prohovnik, I., Smith, G., Sackheim, H. A., Mayeux, R., \& Stern, Y. 1989. Gray-matter degeneration in presenile Alzheimer's disease. Annals of Neurology, 25, 117-124.

Risberg, J., \& Gustafson, L. 1988. Regional cerebral blood flow in psychiatric disorders. In S. Knezevic, V. A. Maximillian, Z. Mubrin, l. Prohovnik, \& J. Wade (Eds.), Handbook of regional cerebral blood flow. Hillsdale, NJ: Erlbaum. Pp. 219-240.

Ryan, J. J., \& Paolo, A. M. 1989. Frequency of occurrence of a WAIS dementia pattern in schizophrenia and bipolar affective disorder. The Clinical Neuropsychologist, 3 , $45-48$.

Satz, P., Hynd, G. W., D’Elia, L., Daniel, M. H., Van Gorp, W., \& Connor, R. 1990. A WAIS-R marker for accelerated aging and dementia, Alzheimer's type?: Base rates of the Fuld formula in the WAIS-R standardization sample. Journal of Clinical and Experimental Neuropsychology, 12, 759-765. 
Satz, P., Hynd, G. W., D'Elia, L., Van Gorp. W. G., Ledbetter, M., \& Connor, R. 1987. A WAIS-R marker for accelerated aging and dementia. Alzheimer's type? Journal of Clinical and Experimental Neuropsychology, 11, 86-87.

Satz, P., Van Gorp, W. G., Soper, H. V., \& Mitrushina, M. 1987. WAIS-R marker for dementia of the Alzheimer's type? An empirical and statistical induction test. Journal of Clinical and Experimental Neuropsychology. 9, 767-774.

Schroeder, T., Holstein, P., Lassen, N. A., \& Engell, H. C. 1986. Measurement of cerebral blood flow by intravenous xenon-133 technique and a mobile system. Neurological Research, 8, 237-242.

Shaw, T.. Meyer, J. S.. Mortel, K., Cutaia, M., Sakai, F., Yamaguchi, F., \& Amamoto, M. 1979. Effects of normal aging, sex, and risk factors for stroke on regional cerebral blood flow (rCBF) in normal volunteers. Acta Neurologica Scandanavica (Suppl.), 60, 462-463.

Stern, Y., Alexander, G. E., Prohovnik, I., \& Mayeux, R. 1992. Inverse relationship between education and parietotemporal perfusion deficit in Alzheimer's disease. Annals of Neurology, 32, 371-375.

Tuokko, H., \& Crockett, D. 1987. Central cholinergic deficiency WAIS profiles in a nondemented aged sample. Journal of Clinical and Experimental Neuropsychology.9, 225-227.

Wechsler, D. 1981. Wechsler Adult Intelligence Scale-Revised manual. Cleveland, OH: The Psychological Corporation, Harcourt Brace Jovanovich. 